\title{
Cardiac Rehabilitation of a Patient With an Advanced Dilated Cardiomyopathy: A Case Report
}

\author{
Chul Kim, MD, PhD, Hee Eun Choi, MD, Byeong-Ju Lee, MD \\ Department of Rehabilitation Medicine, Inje University Sanggye Paik Hospital, \\ Inje University College of Medicine, Seoul, Korea
}

The dilated cardiomyopathy is the common type of cardiomyopathy, and its distinctive characteristic is the systolic dysfunction. Not many reports were issued about the efficacy of cardiac rehabilitation in patients with an advanced dilated cardiomyopathy until yet. A 50-year-old man who was diagnosed with dilated cardiomyopathy with congestive heart failure was admitted to the emergency room after a sudden collapse and a ventricular fibrillation was presented in the actual electrocardiogram. After three months, the patient participated in an 8-week cardiac rehabilitation program with electrocardiogram monitoring for 50 minutes per session at five times per week. The maximal oxygen consumption improved from 13.5 to $19.4 \mathrm{~mL} / \mathrm{kg} / \mathrm{min}$ during this time. At 3.9 metabolic equivalents, the myocardial oxygen demand decreased from 21,710 to $12,669 \mathrm{mmHg} \cdot \mathrm{bpm}$ and the Borg's scale of perceived exertion decreased from 15 to 9 . The left ventricular ejection fraction improved from $14 \%$ to $19 \%$. So in this case report will be presented a patient after a successful cardiac rehabilitation program. Before this the patient suffered from a much more advanced dilated cardiomyopathy and was resuscitated from cardiac arrest.

Keywords Heart arrest, Rehabilitation, Dilated cardiomyopathy

\section{INTRODUCTION}

Dilated cardiomyopathy (DCMP) is one of the most common types of cardiomyopathy and patients suf-

Received May 16, 2013; Accepted August 19, 2013

Corresponding author: Byeong-Ju Lee

Department of Rehabilitation Medicine, Inje University Sanggye Paik Hospital, Inje University College of Medicine, 1342 Dongil-ro, Nowon-gu, Seoul 139-707, Korea

Tel: +82-2-950-1134, Fax: +82-2-935-3076, E-mail: s3163@paik.ac.kr

(c) This is an open-access article distributed under the terms of the Creative Commons Attribution Non-Commercial License (http://creativecommons. org/licenses/by-nc/3.0) which permits unrestricted noncommercial use, distribution, and reproduction in any medium, provided the original work is properly cited.

Copyright (C) 2014 by Korean Academy of Rehabilitation Medicine fering from DCMP show an increase in the myocardial mass and volume. The myocardial wall gradually becomes thinner and stretches and so the left ventricular (LV) function deteriorates [1]. There are many causes for its occurrence and the diagnosis idiopathic DCMP will be given when the underlying pathology cannot be determined [2]. A 50\% 5-year survival rate after diagnosis is reported, as patients often develop a progressive congestive heart failure and complications, such as thromboembolic conditions and arrhythmias [3]. The prognosis is usually bad when the following conditions are given: 1) the LV function is poor with less than $25 \%$ of left ventricular ejection fraction (LVEF), 2) LV enlarge- 
ment, 3) conduction block and arrhythmia are present, and/or 4) the $\mathrm{VO}_{2} \max$ is low [2]. It is well-known a cardiac rehabilitation (CR) program can improve the patients' quality of life, the exercise capacity and the activities of daily living [4-6]. Recently, it has been reported in a study the exercise training (ET) can improve the heart function and motor skills in DCMP patients [7]. However, in this study were targeted only those patients with a mild or moderate disease course (mean LVEF, 39\%) and with a stabilization over 6 months. Not many cardiac rehabilitation studies were presented yet where DCMP patients with advanced disease courses were observed. We report about a case of a successful CR for a patient suffering from a severe advanced DCMP after cardiac arrest resuscitation.

\section{CASE REPORT}

A 50-year-old male school administrator was diagnosed with DCMP with congestive heart failure. He received medical treatment since 1996 and his LVEF was $20 \%$ at the reported time.

On December 12, 2011, the patient was admitted to the emergency room due to a sudden collapse and mentality loss. At the time of initial evaluation, the patient was still unconscious and the electrocardiogram (ECG) showed a ventricular fibrillation. A sinus rhythm returned after 40 minutes of cardiopulmonary resuscitation with defibrillation and intravenous injections of epinephrine and amiodarone. The patient received post-resuscitation care including therapeutic hypothermia, mechanical ventilation, and a tracheostomy in an intensive care unit. After three months, the patient got his consciousness back but his LVEF was $14 \%$ only.

On April 8, 2012, the patient was transferred to the department of rehabilitation medicine where he was provided with $\mathrm{CR}$. The cognitive function measured with the Mini-Mental State Evaluation (MMSE) score was declined to 7 . The limbs were measured to be fair grade in a manual muscle test and the functional level was measured to be 71 at the Functional Independence Measure score. Thus, the patient needed moderate help in self-care, mobility, communication, and social cognition. The existing dysphagia persisted and a Levin tube was inserted therefore. Although the patient was capable to respire spontaneous the T-tube remained due to the dysphagia and the weak power for coughing and sputum expectoration. An
ET monitored with ECG with 1.8 metabolic equivalents (METs) was started and the intensity was gradually increased. The first symptom limited exercise tolerance test (ETT) was conducted at the third week of CR (Fig. 1A). The exercise intensity was increased by 0.5 METs every three minutes according to the manual protocol. After 14 minutes, the ETT was terminated by patient's request due to a dyspnea. At that time, the $\mathrm{VO}_{2}$ max was $13.5 \mathrm{~mL} /$ $\mathrm{kg} / \mathrm{min}$ and the exercise ECG showed frequent premature ventricular contractions. However, the hemodynamic response was acceptable without chest pain. The respiratory exchange ratio was 1.14 . The patient participated in a CR program for 8 weeks in this hospital which included an ECG monitored ET for 50 minutes per session at five times per week (Fig. 1B). Subsequently, a follow-up ETT was done using the same manual protocol after the completion of the CR program. The test was stopped after 28 minutes at the maximal heart rate (HR) of $178 \mathrm{bpm}$. The $\mathrm{VO}_{2}$ max improved from 13.5 to $19.4 \mathrm{~mL} / \mathrm{kg} / \mathrm{min}$ ( $43 \%$ increase) and the myocardial oxygen demand at the submaximal level decreased from 21,710 (3.9 METs) to 12,669 (3.9 METs) mmHg.bpm. The peak myocardial oxygen demand was 19,936 (5.5 METs) mmHg.bpm. The Borg's scale of perceived exertion at 3.9 METs decreased from 15 to 9 (Table 1). A follow-up transthoracic echocardiogram (TTE) showed a slight improvement of the LVEF from $14 \%$ to $15 \%$ (Table 2 ).
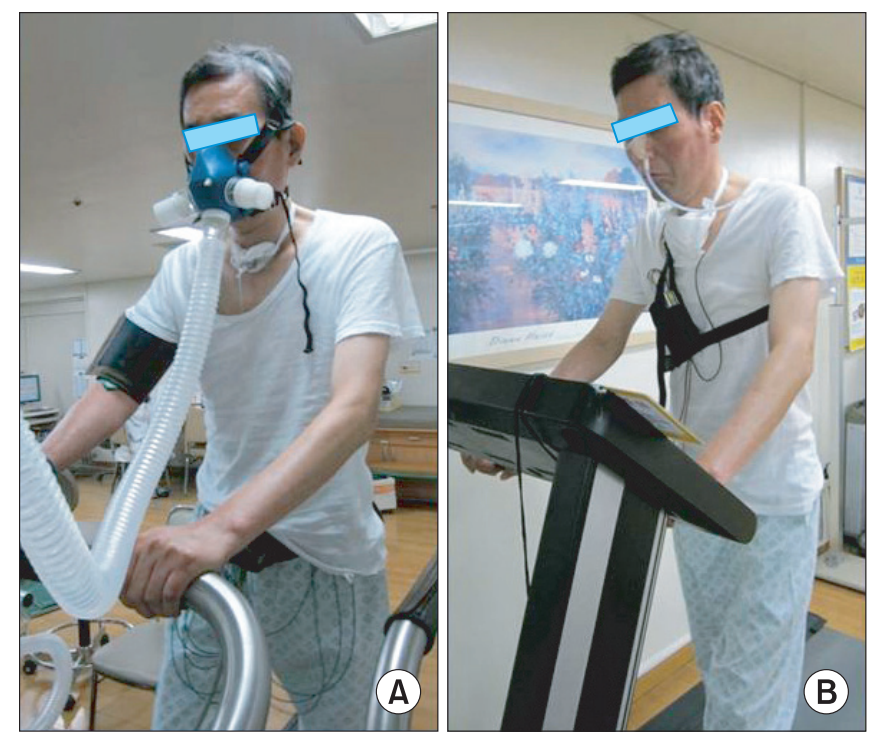

Fig. 1. (A) The symptom limited exercise tolerance test. (B) The electrocardiogram monitored the exercise training. 
Table 1. Hemodynamic results of symptom limited exercise test

\begin{tabular}{lcccc}
\hline & Apr 26, 2012 & \multicolumn{2}{c}{ Jun 13, 2012 } & Dec 26, 2012 \\
\hline Protocol & Manual & \multicolumn{2}{c}{ Manual } & Modified Bruce \\
METs & 3.9 & 3.9 & 5.5 & 5.6 \\
Maximal heart rate (bpm) & 167 & 123 & 178 & 151 \\
Maximal systolic BP (mmHg) & 130 & 103 & 112 & 114 \\
Maximal diastolic BP (mmHg) & 80 & 55 & 57 & 70 \\
\hline Rate pressure product (mmHg·bpm) & 21,710 & 12,669 & 19,936 & 15,015 \\
\hline Borg's scale of perceived exertion $^{\text {a) }}$ & 15 & 7 & 11 & 17 \\
VO $_{2}$ max (mL/kg/min) & 13.5 & - & 19.4 & 19.5 \\
Respiratory exchange ratio $_{\text {Total exercise time }}$ & 1.16 & - & 1.14 & 0.98 \\
\hline
\end{tabular}

METs, metabolic equivalents; bpm, beats per minute; $\mathrm{BP}$, blood pressure; $\mathrm{VO}_{2} \mathrm{max}$, maximal oxygen consumption.

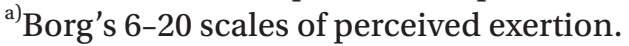

Table 2. Serial follow-up of echocardiogram

\begin{tabular}{|lccc}
\hline & $\begin{array}{c}\text { Mar 7, 2012 } \\
\text { Pre-ET }\end{array}$ & $\begin{array}{c}\text { Jun 4, 2012 } \\
\text { 8 weeks post-ET }\end{array}$ & $\begin{array}{c}\text { Sep 21, 2012 } \\
\text { After 3 months }\end{array}$ \\
\hline Ejection fraction (\%) & 14 & 15 & 19 \\
\hline LV diastolic distance (mm) & 8.01 & 8.17 & 8.20 \\
LV systolic distance (mm) & 7.39 & 7.48 & 7.34 \\
Stroke volume (mL) & 45 & 64 & 78.9 \\
Diastolic function & & & 0.98 \\
E (m/s) & 0.80 & & 0.07 \\
E' (m/s) & 0.03 & 0.37 \\
A (m/s) & 0.08 & 2.65 \\
\hline E/A & 1.90 & & 14.00 \\
\hline E/E' & 26.67 & & 118 \\
\hline Deceleration time (ms) & 88 & 0.73 \\
Thickness of interventricular septum (cm) & 0.90 & 0.82 & 415 \\
LVEDV (mL) & 321 & 427 & 32 \\
\hline Wall motion score & 32 & 32 & \\
\hline
\end{tabular}

ET, exercise training; LV, left ventricular; LVEDV, left ventricular end-diastolic volume. E, the early ventricular filling velocity; E', peak annuls velocity during early filling; A, the late ventricular filling velocity; E/A, the ratio of the early (E) to late (A) ventricular filling velocities; E/E', the ratio of the early ventricular filling velocity to peak annuls velocity during early filling.

${ }^{\text {a)}}$ LVEDV = stroke volume / ejection fraction [9].

The patient's MMSE improved from 7 to 13 and the activities of daily living could be independently performed almost. The dysphagia therapy and the swallowing functional electrical stimulation were continued. No aspiration or penetration was shown during a videofluoroscopic swallowing study and the patient could sufficiently expectorate by himself. L-tube and T-tube were success- fully removed.

The patient performed an outpatient CR program with three times a week after the discharge from the department of rehabilitation. On December 26, 2012, a followup ETT was performed with the modified Bruce protocol for the first time. The test was stopped after 13 minutes due to patient's request at the maximal HR of $191 \mathrm{bpm}$ 
and the $\mathrm{VO}_{2}$ max was $19.5 \mathrm{~mL} / \mathrm{kg} / \mathrm{min}$. The recent LVEF was $19 \%$.

The patient received cardiac resynchronization therapy under the use of biventricular pacing according to the latest heart failure treatment guidelines [8] and received also outpatient CR for additional 3 months. Together with his wife he is preparing to return to his previous job.

\section{DISCUSSION}

The CR program is well-known for its safety and effectiveness to improve the exercise capacity and the quality of life in patients with heart failure [4-6]. Holloway et al. [7] performed an 8-week CR program on patients with mild to moderate DCMP. After this 8-week training period, the LVEF was improved secondary to the increase of the left ventricular end-diastolic volume (LVEDV). It means either the diastolic function was improved or the intestinal/splenic venoconstriction was enhanced and the latter would be consistent with an improved autonomic function which follows the ET. Interestingly, the improvement of LVEF was more dominant in patients with a sedentary state. But the ET does not alter the cardiac high-energy phosphates during rest or physical exercise. This may suggest exercising leads not to a positive remodeling, but may improve the general reconditioning of the diseased myocardium or other skeletal muscles. Sullivan et al. [9] performed CR programs with 16 to 24 weeks with 12 patients with LV dysfunction. The peak oxygen consumption statistically increased but the maximal cardiac output showed an increasing trend only $(\mathrm{p}=0.13)$. There was no difference found in the central hemodynamic results, such as rest and exercise measurements of LVEF. But the trend seemed to be due to peripheral adaptation like an increase in the arteriovenous oxygen difference.

In this present case, the exercise capacity was improved after the ET. The LVEDV was improved from 321 to 415 $\mathrm{mL}$ and lead to an improvement of LVEF from $14 \%$ to $19 \%$. In the follow-up TTE, the cardiac wall motion was generally similar, but the thickness of the interventricular septum and E/E' were reduced. It suggests that the diastolic function slightly improved over the time of the CR. Depending on the results of TTE, ETT and in accordance to previous literature, the ET effects on the DCMP may be related to the reconditioning of the cardiac and skeletal muscles and peripheral adaptation although the phosphocreatinine to ATP ratio and arteriovenous oxygen differences were not tested.

The ECG monitor showed frequent single premature ventricular contractures (PVC) about $20 \mathrm{bpm}$ during the early period of ET. There was no life-threatening event, such as ventricular arrhythmia or hemodynamic instability, after 4 weeks of training due to a gradual reduction of PVC frequency.

In Korea, CR is not actively performed for DCMP patients with a severe heart failure currently. The authors of this report safely performed a CR program on a patient with an advanced DCMP and experienced that the CR was effective in the improvement of exercise capacity and quality of life. Further studies will be needed in the near future to show the effects of CR on the heart function itself and to suggest a CR program guideline for patients with an advanced DCMP.

\section{CONFLICT OF INTEREST}

No potential conflict of interest relevant to this article was reported.

\section{REFERENCES}

1. Schoen F. The heart. In: Kumar V, Abbas AK, Fausto N, editors. Robbins and Cotran pathological basis of disease. 7th ed. Philadelphia: Elsevier; 2005. p. 555-618.

2. Luk A, Ahn E, Soor GS, Butany J. Dilated cardiomyopathy: a review. J Clin Pathol 2009;62:219-25.

3. Grogan M, Redfield MM, Bailey KR, Reeder GS, Gersh BJ, Edwards WD, et al. Long-term outcome of patients with biopsy-proved myocarditis: comparison with idiopathic dilated cardiomyopathy. J Am Coll Cardiol 1995;26:80-4.

4. Stolen KQ, Kemppainen J, Ukkonen H, Kalliokoski KK, Luotolahti M, Lehikoinen P, et al. Exercise training improves biventricular oxidative metabolism and left ventricular efficiency in patients with dilated cardiomyopathy. J Am Coll Cardiol 2003;41:460-7.

5. Belardinelli R, Georgiou D, Cianci G, Purcaro A. Randomized, controlled trial of long-term moderate exercise training in chronic heart failure: effects on functional capacity, quality of life, and clinical outcome. Circulation 1999;99:1173-82. 
6. O'Connor CM, Whellan DJ, Lee KL, Keteyian SJ, Cooper LS, Ellis SJ, et al. Efficacy and safety of exercise training in patients with chronic heart failure: HFACTION randomized controlled trial. JAMA 2009;301: 1439-50.

7. Holloway CJ, Dass S, Suttie JJ, Rider OJ, Cox P, Cochlin LE, et al. Exercise training in dilated cardiomyopathy improves rest and stress cardiac function without changes in cardiac high energy phosphate metabo- lism. Heart 2012;98:1083-90.

8. Abraham WT, Fisher WG, Smith AL, Delurgio DB, Leon AR, Loh E, et al. Multicenter InSync Randomized Clinical Evaluation: cardiac resynchronization in chronic heart failure. N Engl J Med 2002;346:1845-53.

9. Sullivan MJ, Higginbotham MB, Cobb FR. Exercise training in patients with severe left ventricular dysfunction: hemodynamic and metabolic effects. Circulation 1988;78:506-15. 